\title{
Solidificación-Estabilización de Cromo, Níquel y Plomo en una Matriz Sólida de Hormigón Fabricada con Cemento Portland
}

René A. Lara-Díaz ${ }^{1,2}$ y Rosa M. Melgoza-Alemán ${ }^{2}$

(1) Universidad de las Américas Puebla, Depto. Ing. Química y Alimentos, Ex Hacienda Santa Catarina Mártir s/n, 72820, San Andrés Cholula, Puebla-México (e-mail: renea.lara@udlap.mx)

(2) Univ. Autónoma del Estado de Morelos, Fac. Ciencias Químicas e Ingeniería, Centro de Inv. en Ing. y Ciencias Aplicadas, Av. Universidad 1001, Col. Chamilpa, 62209 Cuernavaca, Morelos-México

\begin{abstract}
Resumen
Se validó la técnica de cementación como alternativa para la solidificación-estabilización de residuos sintéticos de metales pesados, cromo, níquel y plomo, usando probetas sólidas de hormigón de cemento Portland. El proceso de cementación se realiza a partir de una mezcla base para obtener hormigón con resistencia a la compresión de $29.4 \mathrm{~N} / \mathrm{mm}^{2}$, los metales fueron incorporados como sales metálicas en el agua de amasado. Se realizaron pruebas de resistencia a la compresión, lixiviación de metales por la prueba PECT con absorción atómica y se calculó la eficiencia de retención. El hormigón fabricado con cemento Portland es adecuado para su uso en la solidificaciónestabilización de níquel y plomo a una concentración máxima de $0.43 \%$ para níquel y 1.94\% para plomo.
\end{abstract}

Palabras clave: hormigón, solidificación-estabilización, cemento Portland, metales pesados

\section{Solidification-Stabilization of Chromium, Nickel and Lead in a Concrete Solid Matrix of Portland Cement}

\begin{abstract}
The technique of cementation was validated as an alternative method for the solidificationstabilization of heavy metal synthetic wastes, chromium, nickel and lead, using concrete solid cylinders made of Portland cement. The cementation process takes place starting with a concrete mixture base with compressive strength of $29.4 \mathrm{~N} / \mathrm{mm}^{2}$. Metals were incorporated as metallic salts in the mixing water. Tests of compressive strength, leaching of metals by PECT with atomic absorption were performed and retention efficiencies were calculated. Concrete made with Portland cement is appropriate for the solidification-stabilization of nickel and lead, with maximum concentrations of $0.43 \%$ of nickel and $1.94 \%$ of lead in concrete.
\end{abstract}

Keywords: concrete, solidification-stabilization, Portland cement, heavy metals, leaching 


\section{INTRODUCCIÓN}

El problema de la contaminación del medio ambiente se puede establecer en varias ramas, las aguas residuales y el manejo de los residuos son dos de los tipos que pueden ser atendidos con la metodología de solidificación-estabilización (SE) (Tchobanoglous et al., 2003). La solidificaciónestabilización es un proceso que se ha utilizado desde finales de los 80's para atrapar contaminantes limitando la movilidad y también disminuyendo su potencial de afectación al medio ambiente y que estos residuos puedan ser dispuestos en rellenos sanitarios (Culliname et al., 1986) y en particular en Europa donde no se reciben sin pretretamiento (Aubert et al., 2006).

De acuerdo a la Agencia de Protección Ambiental de los Estados Unidos la SE ha sido utilizada en el $24 \%$ de los casos de remediación de sitios contaminados con residuos peligrosos bajo control federal (USEPA, 2004). El proceso de SE ha sido considerado en esos casos, la mejor tecnología disponible demostrable para la remediación de sitios. Sin embargo, no es una tecnología que se encuentre totalmente desarrollada (Paria y Yuet, 2006) y Glasser (1997) menciona que muchos aspectos de esta metodología permanecen poco entendidos y mal cuantificados.

Existen varias técnicas de SE como son: la absorción en materiales para el posterior manejo de materiales, que comúnmente se ha utilizado para control de derrames; la coagulación y precipitación para insolubilizar sustancias y pasarlas a fase sólida; la cementación que usa cemento portland o puzolánico para crear monolitos de hormigón que contengan principalmente contaminantes inorgánicos; la plastificación que forma estructuras termoplásticas que por polimerización atrapan compuestos orgánicos así como la vitrificación que mediante la producción de vidrio atrapa contaminantes inorgánicos y degrada orgánicos por oxidación (USEPA, 2004).

La cementación usando cemento portland presenta posibilidades para el atrapado de compuestos orgánicos e inorgánicos (Paria y Yuet, 2006). Existen reportes de autores que han probado la utilización de la SE con cemento portland como una aplicación exitosa para el tratamiento de residuos tóxicos con metales (Al-Tabbaa y Prose, 1996), residuos de la industria siderúrgica (Silveira et al., 2003), cenizas de incineración (Aubert, et al., 2006), cenizas de madera (Udoeyo, et al., 2006) o lodos industriales (Carmalin y Swaminathan, 2005). Se han realizado trabajos de tratamiento de muestras de suelos contaminados usando cemento portland como aglomerante para la retención de cromo, plomo y cobre (Polettini et al., 2004). La inmovilización de compuestos orgánicos ha sido más difícil debido a que interfieren en el proceso de formación del hormigón (Paria y Yuet, 2006). La tendencia de la inclusión de orgánicos ha cambiado, porque ha servido para reducir la velocidad de la reacción de hidratación para mejorar el manejo comercial del cemento con poca afectación en la retención de metales como plomo y zinc (Zhang et al., 2008). Otros trabajos han profundizado en el estudio de la estructura microscópica del hormigón (Klich et al., 1999) o en la modelación de la solubilidad de los metales del hormigón (Fernández-Olmo et al., 2007).

La SE con cemento portland presenta ventajas importantes para el tratamiento de residuos peligrosos con metales como son: requerimientos técnicos de personal bajos, bajo costo de producción, estabilidad estructural del hormigón a largo plazo, alta resistencia a la biodegradación, relativamente baja permeabilidad del hormigón, la no toxicidad los materiales usados y la composición estandarizada del cemento obtenido en distintos lugares (Shi y Spence, 2004). Sin embargo, también se han reportado algunas desventajas en su utilización. Pellegrini et al. (2006), indica que la carbonatación del hormigón produce una desorción de los metales en el tiempo. Klich et al. (1999), cuestionan la integridad de las piezas de hormigón en un estudio de envejecimiento.

El proceso de cementación involucra la participación de varios fenómenos que en forma conjunta permiten la retención de los metales en el estrato sólido: la absorción de líquidos con metales en solución que disminuyen la capacidad de campo del residuo a tratar, la precipitación química en un ambiente alcalino del hormigón ya que se forman hidróxidos metálicos insolubles a valores de $\mathrm{pH}$ alrededor de 10, la fijación por reacción química de los metales con los productos de hidratación del hormigón, la adsorción de los metales en las superficies de los productos de hidratación así como la encapsulación física de los metales por la formación de la fase sólida del hormigón (Glasser, 1997). La cementación en particular consiste en la formación de compuestos insolubles resultado de las 
reacciones de hidratación del cemento donde se producen hidróxidos de los metales por el medio alcalino de reacción (Silveira et al., 2003). Mijno et al. (2004), mencionan que se obtienen varias fases sólidas como porlandita (hidróxido de calcio), hidrato de silicato de calcio, ettringita $\left(\mathrm{Ca}_{6} \mathrm{Al}_{2}\left(\mathrm{SO}_{4}\right)_{3} \cdot 26 \mathrm{H}_{2} \mathrm{O}\right)$ y monosulfato $\left.\left[\mathrm{Ca}_{2}(\mathrm{Al}, \mathrm{Fe}) \mathrm{OH} 6\right]_{2}\left(\mathrm{SO}_{4}\right) \cdot x \mathrm{H}_{2} \mathrm{O}\right]$ durante el proceso de hidratación del cemento; y que los metales se incorporan por sustitución de los iones de calcio y aluminio principalmente así como por absorción y mezclado.

La característica ambiental importante para establecer la viabilidad para la disposición de los productos de SE en rellenos sanitarios o la remediación de suelos es la prueba de lixiviación denominada prueba de extracción de compuestos tóxicos (PECT) basada en la norma NOM-053SEMARNAT-1993 (2003), prueba equivalente a la TCLP (toxic characteristic leaching procedure) que es un criterio internacional aceptado para determinar la no peligrosidad de una muestra de residuo. Para valores de concentración de metales pesados en el lixiviado PECT mayores a $5.0 \mathrm{mg} / \mathrm{l} \mathrm{se}$ tipifica el material de origen como un residuo o material peligroso por toxicidad de acuerdo a la norma NOM-052-SEMARNAT-2005 (2006). La lixiviación de metales se ha considerado dependiente del pH (Malviya y Chaudhary, 2006), se han realizado estudios de lixiviación de metales en hormigón con soluciones de extracción a pH de 4, 7 y 9, mostrando que a valores inferiores a 5 se tienen las concentraciones con mayor lixiviación de metales (Fernández-Olmo et al., 2003). Resultados similares se reportan por Van der Sloot (2002) en un rango de pH entre 3 y 13 unidades.

La característica constructiva importante del hormigón es la resistencia a la compresión. Conforme a las especificaciones de construcción NMX-C-414-ONNCCE-2004 (2004), se establece que 9.8 $\mathrm{N} / \mathrm{mm}^{2}$ de resistencia a la compresión del hormigón es adecuada para construcción de pisos, firmes y banquetas y $14.7 \mathrm{~N} / \mathrm{mm}^{2}$ para trabes, dalas y cadenas. Zhang et al. (2008), ha caracterizado hormigón con esta técnica y Silveira et al. (2003), citan que son muchos los factores que afectan la formación y resistencia a la compresión de la matriz de hormigón como son: calidad del cemento, agua, agregados, aditivos, tiempo de fraguado, entre otros.

La propuesta de este estudio se enfoca a evaluar la efectividad del método solidificaciónestabilización por cementación usando cemento portland para la retención de metales pesados (cromo, níquel y plomo). Los metales se incorporan disueltos en agua a distintas concentraciones, como muestras sintéticas de aguas residuales de la industria de acabado metálico, para analizar las tendencias de lixiviación, resistencia a la compresión y eficiencia de retención en función del contenido de metal en el hormigón. Los resultados se comparan con el criterio de no peligrosidad para ser dispuesto en rellenos sanitarios como residuo no peligroso y el criterio de resistencia a la compresión suficiente para aplicaciones comerciales de baja especificación y se establecen valores de concentración que satisfacen ambos criterios.

\section{MATERIALES Y MÉTODOS}

Cemento portland: El cemento portland utilizado es producido comercialmente a partir de clinker y yeso. De acuerdo a la forma de clasificación de la NMX-C-414-ONNCCE-2004 (2004), como cemento portland ordinario CPO, resistente a los sulfatos RS, con una resistencia a la compresión máxima de $40 \mathrm{~N} / \mathrm{mm}^{2}$.

Arena: La arena usada es un agregado granular fino de tamaño promedio nominal de $2.1 \mathrm{~mm}$, determinado por prueba de granulometría con tamices, con composición es principal de cuarzo, mica y feldespato. La arena utilizada para es de origen aluvial, sin trituración, libre de polvo, materia orgánica y otras sustancias objetables; y se adquirió en una empresa de materiales de construcción al mayoreo, su denominación comercial es "arena de río".

Grava: La grava usada es obtenida de trituración de roca, con una granulometría en el rango de 12.7 $\mathrm{mm}$ a $20.0 \mathrm{~mm}$, con composición principal de minerales de caliza, granito, dolomita, basalto, arenisca, cuarzo y cuarcita. La arena se adquirió en una empresa de materiales de construcción al mayoreo y su nombre comercial es "grava de construcción".

Información Tecnológica Vol. - 20 N¹ - 2009 
Agua: El agua utilizada para la preparación de soluciones de metales es agua destilada desionizada grado reactivo con especificación de conductividad de $0.8 \mu \mathrm{S} / \mathrm{cm}, \mathrm{pH}$ de 6.5 unidades, sodio y dureza de $0 \mathrm{mg} / \mathrm{l}$. El agua restante requerida para la fabricación de probetas de hormigón es agua potable obtenida de la red de abastecimiento municipal con $0 \mathrm{mg} / \mathrm{l}$ de cloro y cumplió la norma NOM-127SSA1-1994 (2000).

Sales metálicas: Los metales del estudio son cromo VI, níquel II y plomo II. Las sales utilizadas para la preparación de las soluciones son sales grado reactivo de trióxido de cromo, cloruro de níquel y nitrato de plomo. La preparación de las soluciones de la Tabla 1 se realiza con sales secas y considerando el grado de hidratación y pureza reportado por fabricante. La formulación del hormigón es en porcentaje de sal para mantener una relación de producción constante, sin embargo, los resultados se expresan en porcentaje de metal en el hormigón para hacer comparativos los resultados.

Hormigón: La preparación base de hormigón fue fabricada con cemento portland con una dosificación de materiales para tener una resistencia a la compresión de $29.4 \mathrm{~N} / \mathrm{mm}^{2}$. La preparación base consistió de cemento portland CPO $18.1 \%$, arena $30.6 \%$, grava $43.2 \%$ y agua $8.1 \%$. La cantidad de agua se denomina agua de amasado y es la cantidad máxima de agua permisible; de esta manera se preparan las soluciones de sales metálicas con un máximo del $50 \%$ del agua de amasado usando agua potable y el porcentaje restante se incorpora sin metales para tener la matriz de composición que se presenta en la Tabla 1. Los porcentajes de sales son de cada sal en forma independiente y no se probaron interacciones de mezclas de sales.

Tabla 1: Matriz de composición de hormigón con diferente contenido de sales de los metales

\begin{tabular}{|c|c|c|c|c|}
\hline Componente & \multicolumn{4}{|c|}{ Porcenatje de composición } \\
\hline Sal metálica & 1.00 & 3.00 & 5.00 & 10.00 \\
\hline Agua & 8.07 & 7.90 & 7.74 & 7.33 \\
\hline Cemento & 17.93 & 17.57 & 17.21 & 16.30 \\
\hline Arena & 30.25 & 29.64 & 29.02 & 27.50 \\
\hline Grava & 42.75 & 41.89 & 41.03 & 38.87 \\
\hline Total & 100 & 100 & 100 & 100 \\
\hline
\end{tabular}

Fabricación de probetas de hormigón: Se fabricaron probetas de hormigón por duplicado, realizando el vaciado manual en capas de 4"; se usó una varilla metálica de acero inoxidable (redondo 5/8" y longitud de 24") para eliminar aire por aplicación 25 golpes por sección, aplicando 3 secciones de hormigón en húmedo ya incluyendo los metales en la solución de amasado. Los moldes usados tienen una proporción de longitud 2:1 y las dimensiones son de 6" de diámetro por 12" de altura. Las probetas están fabricadas de dos medias cañas en acero inoxidable que permanecen unidas por 48 horas y se produce el fraguado de las probetas. Posteriormente se realiza la curación de las probetas dejando reposar en cuarto con humedad controlada hasta el cumplimiento de 28 días.

Resistencia a la compresión: La determinación de resistencia a la compresión consiste en la determinación de la fuerza que se aplica sobre la superficie circular de las probetas curadas. A los cilindros se les realiza previamente a la determinación un afinado de la superficie aplicando azufre líquido y dejando enfriar por 10 minutos. La prueba de resistencia a la compresión se realizó por duplicado en un equipo Tinus-Olsen y se determinó la fuerza aplicada hasta que cede la estructura de hormigón, el área se determina por la medición con Vernier de la sección transversal del cilindro de la probeta. La lectura de fuerza se obtuvo en $\mathrm{kg}_{\mathrm{f}}$ y el área en $\mathrm{cm}^{2}$, se calcula la resistencia a la compresión como la fuerza aplicada entre el área y se convierte a unidades de $\mathrm{N} / \mathrm{mm}^{2}$.

Prueba de lixiviación PECT: La prueba de lixiviación PECT consiste en la extracción en medio ácido de la muestra de hormigón fracturado de la prueba de resistencia a la compresión. La determinación se basa en la NOM-053-SEMARNAT-1993 (2003). El procedimiento consiste en extracción con reactivo de extracción RE1 o RE2 en función del pH. Se selecciona el reactivo de extracción al poner $5 \mathrm{~g}$ de muestra sólida con diámetro de $1 \mathrm{~mm}$ o menor, con $96.5 \mathrm{ml}$ de agua destilada desionizada y 
se agita por 5 minutos, si el pH es menor a 5 unidades se utiliza el reactivo de extracción RE1. A un $\mathrm{pH}$ superior a 5 , se añaden $3.5 \mathrm{ml}$ de $\mathrm{HCl} 1 \mathrm{~N}$ se calienta a $50^{\circ} \mathrm{C}$ por 10 minutos, se deja enfriar a temperatura ambiente si el pH es menor a 5 unidades se utiliza RE1 o si el pH es superior o igual a 5 unidades se usa RE2. La prueba de lixiviación se determina con $100 \mathrm{~g}$ de muestra sólida con diámetro menor a $1 \mathrm{~mm}$, se agrega el reactivo de extracción correspondiente en proporción de 20 veces el peso de la muestra, la muestra se pone en un matraz Erlenmeyer sellado con película de teflón a una velocidad de agitación de $30 \pm 2$ rpm, realizado en un agitador orbital y la agitación se mantiene por un tiempo de $18 \pm 2 \mathrm{~h}$, tiempo después del cual se realiza un filtrado con un sistema de filtración Kitazato, usando filtro de fibra de vidrio para recolectar el extracto. El extracto debe de preservarse a $4^{\circ} \mathrm{C}$, acidificando con ácido nítrico para un $\mathrm{pH}$ inferior a 2 unidades. Los contenedores que vayan a estar en contacto con los extractos para la determinación de metales deberán de ser prelavados con ácido nítrico $1 \mathrm{~N}$. El RE 1 se prepara con $5.7 \mathrm{ml}$ de ácido acético glacial adicionados a $500 \mathrm{ml}$ de agua destilada desionizada, se agregan $64.3 \mathrm{ml}$ de $\mathrm{NaOH} 1 \mathrm{~N}$ y se afora a $1 \mathrm{~L}$, se debe de tener una solución con un pH de 4.93 \pm 0.05 . El RE2 se prepara con $5.7 \mathrm{ml}$ de ácido acético glacial y se afora con agua destilada desionizada a $1 \mathrm{~L}$, la solución debe tener un pH de $2.88 \pm 0.05$ unidades. Esta prueba se realiza por duplicado.

Determinación de metales por absorción atómica: La determinación de metales se realiza por medio de absorción atómica en un equipo Varian SpectrAA 220FS por triplicado, con lámparas de cátodo hueco de un solo elemento y longitudes de onda de $357.9 \mathrm{~nm}$ para cromo, $217.0 \mathrm{~nm}$ para plomo y $232.0 \mathrm{~nm}$ para níquel. Se realizan soluciones para curvas de calibración de concentraciones entre 0.5 a $5.0 \mathrm{mg} / \mathrm{l}$ de metal y son almacenadas en refrigeración a $4^{\circ} \mathrm{C}$ un máximo de 6 meses de acuerdo a la norma NMX-AA-051-SCFI-2001 (2001). Los tubos de ensayo para almacenamiento de estándares de calibración son fabricados en vidrio de borosilicato y tapa de baquelita. La flama del equipo de absorción atómica se realiza por medio de una mezcla de aire y acetileno. El análisis de metales de los extractos se realiza en una sola determinación y por triplicado en un período máximo de 14 días de acuerdo a la norma NOM-053-SEMARNAT-1993 (2003). El método de absorción atómica no distingue estados de oxidación por lo que las determinaciones son como cromo, níquel y plomo totales.

Cálculo de eficiencia de retención de metales: El cálculo de la eficiencia de retención se realiza por medio de un balance de materiales para cada uno de los metales y por cada una de las determinaciones (Yilmaz et al., 2003):

$E_{\mathrm{R}}=\frac{100\left(\mathrm{M}_{\mathrm{MH}}-\mathrm{M}_{\mathrm{ML}}\right)}{\mathrm{M}_{\mathrm{MIH}}}$

donde, $E_{R}=$ Eficiencia retención de metal en el hormigón (\%), $M_{M I H}=$ Masa de metal incorporada en el hormigón y $\mathrm{M}_{\mathrm{ML}}=$ Masa de metal extraída en el lixiviado. El valor de $\mathrm{M}_{\mathrm{MIH}}$ se obtiene de la cantidad de sal utilizada en la preparación de la Tabla 1 para la muestra específica, corregido a fracción de metal por pureza de la muestra, grado de hidratación y tipo de sal. El valor de $\mathrm{M}_{\mathrm{ML}}$ se obtiene de la concentración de metal en el lixiviado por muestra expuesta a lixiviación, el valor se corrige a masa total preparada de hormigón.

Determinación de no-peligrosidad: La determinación de no peligrosidad de una muestra de hormigón se establece si el análisis de metales por absorción atómica de la muestra de lixiviación, posteriormente a las correcciones por dilución y agregado de preservadores tiene valores menores a los presentados en la Tabla 2. De acuerdo a la normativa mexicana se considera una muestra no peligrosa por el criterio de toxicidad.

Precauciones: La exposición a la toxicidad de los metales pesados es un riesgo durante la experimentación y se tomaron las siguientes medidas de seguridad: durante el manejo de las sales metálicas para preparar las soluciones se usaron guantes de latex, mascarilla respiratoria para partículas y restricción de ventilación para evitar suspensión de partículas; durante la fabricación de probetas el uso de guantes de latex para el amasado y llenado de probetas. Los residuales de 
materiales de las pruebas de lixiviación PECT y resistencia a la compresión fueron dispuestos para el manejo controlado de residuos.

Tabla 2: Límites máximos permisibles para los constituyentes tóxicos en el lixiviado PECT

\begin{tabular}{|c|c|c|}
\hline Metal pesado & Límite $(\mathrm{mg} / \mathrm{l})$ & Norma de Referencia \\
\hline Cromo & 5.0 & NOM-052-SEMARNAT-2005 \\
\hline Plomo & 5.0 & NOM-052-SEMARNAT-2005 \\
\hline Níquel & 5.0 & No fijado por la norma pero establecido con mismo valor \\
\hline
\end{tabular}

\section{RESULTADOS Y DISCUSIÓN}

Se fabricaron probetas de hormigón a partir de la composición base establecida en la Tabla 1 , usando soluciones de sales de cromo, plomo, níquel y transcurridos 28 día de fraguado/curado se realizan pruebas de resistencia a la compresión. Se fabricó un testigo sin sales metálicas que presenta una resistencia a la compresión de $29.2 \mathrm{~N} / \mathrm{mm}^{2}$ contra $29.4 \mathrm{~N} / \mathrm{mm}^{2}$ de la formulación de diseño, para validación de la resistencia a la compresión de la fórmula base.

En la Fig. 1 se presentan los resultados de la resistencia a la compresión en función de la incorporación de metales en la formulación del hormigón, se aprecia una disminución de la resistencia a la compresión a incremento de metal en el hormigón. La resistencia a la compresión disminuye rápidamente a baja concentraciones de metal en el hormigón, presenta una inflexión después de la cuál el decrecimiento se vuelve más lento para todos los metales.

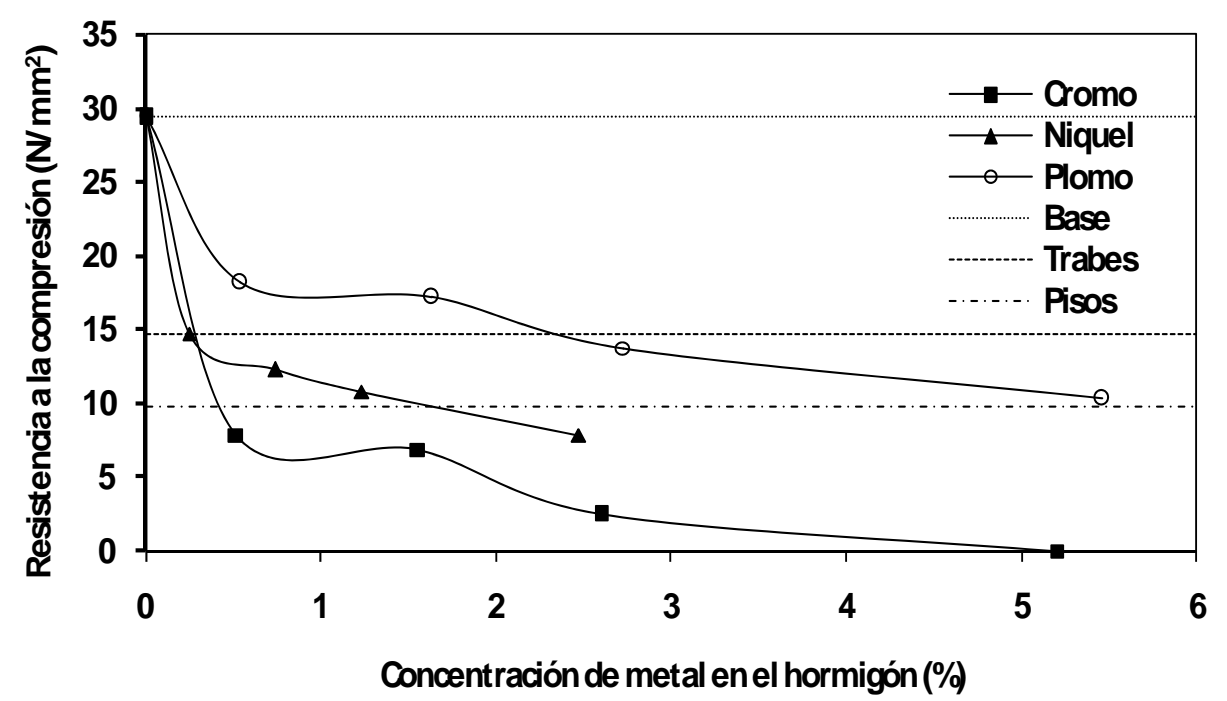

Fig. 1: Resistencia a la compresión de probetas de hormigón usando cemento portland, así como resistencia base, especificaciones constructivas para trabes y pisos

Los límites de resistencia a la compresión para construcción de pisos, firmes y banques $\left(9.8 \mathrm{~N} / \mathrm{mm}^{2}\right)$ y para trabes, dalas y cadenas $\left(14.7 \mathrm{~N} / \mathrm{mm}^{2}\right)$ se toman de las especificaciones de la norma NMX-C414-ONNCCE-2004 (2004). Se observa que para concentraciones de cromo superiores a $5 \%$ la resistencia a la compresión es nula ya que la pieza experimenta un desmoronamiento al momento de vaciado del molde de la probeta a las 48 horas. Así también se puede establecer que para resistencia a la compresión de $14.7 \mathrm{~N} / \mathrm{mm}^{2}$ equivalente a una pérdida del $50 \%$ del valor de diseño, la composición máxima permisible para cromo es de $0.25 \%$, para níquel es de $0.2 \%$ y para plomo es de 2.4\%; para valores de resistencia a la compresión de $9.8 \mathrm{~N} / \mathrm{mm}^{2}$, la composición máxima para cromo es de $0.4 \%$, para níquel es de $1.6 \%$ y para plomo es de $5.7 \%$. Los comportamientos de pérdida de resistencia a la compresión no son lineales y se observa que el cromo es el que presenta menor afinidad en la formación del hormigón y fue el único que en el rango de estudio causó un desmoronamiento total de las piezas al momento de fraguar. 
Las piezas fracturadas obtenidas de la prueba de resistencia a la compresión se utilizaron para la determinación de la prueba de lixiviación PECT. En el caso experimentado se utilizó reactivo dos (RE2) para la extracción de los tres metales del hormigón, el pH de extracción fue de 2.9 unidades. En la Figura 2 se muestra que los valores de concentración de metal en el lixiviado se incrementan en función de la concentración de metal en el hormigón, con una tendencia casi lineal.

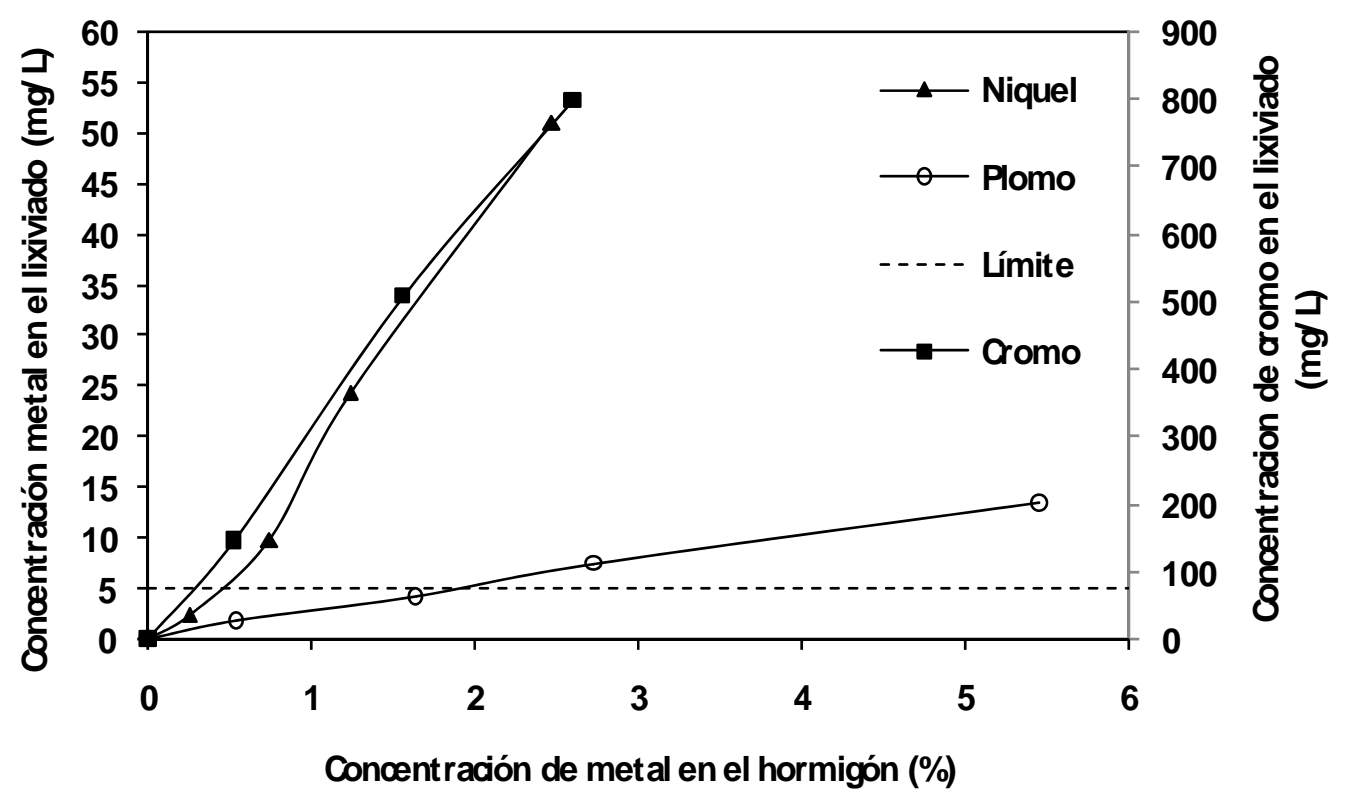

Fig. 2: Concentración de cromo, níquel, plomo y límite de no-peligrosidad en el lixiviado PECT

En este estudio se obtuvo un mejor comportamiento de la cementación con valores de concentración en el lixiviado de $13.4 \mathrm{mg} / \mathrm{l}$ para una concentración en el hormigón de 5.5\%., en tanto que Yilmaz et al. (2003), obtuvieron valores de plomo en el lixiviado de $70 \mathrm{mg} / \mathrm{l}$ a una concentración de $5.75 \%$ de plomo en el hormigón. Para al cromo se observa que los valores de lixiviación son significativamente más altos, $595 \mathrm{mg} / \mathrm{l}$, para una concentración de $2 \%$ de cromo en el hormigón contra los valores de plomo de $5.1 \mathrm{mg} / \mathrm{l}$ y níquel de $39 \mathrm{mg} / \mathrm{l}$ respectivamente. Se observa que el plomo es el elemento con mayor afinidad al hormigón ya que la concentración en el lixiviado es la menor en el rango de estudio, a continuación se encuentra el níquel y el cromo es el que presenta menor afinidad.

Las concentraciones máximas permisibles de metal en el hormigón para satisfacer el criterio nopeligrosidad se obtienen de la intersección del valor máximo permisible de la norma de $5.0 \mathrm{mg} / \mathrm{l}$ (Tabla 2) y las curvas de las Fig. 2, las cuales son para cromo de $0.02 \%$, para níquel de $0.43 \%$ y para plomo de 1.94\%. Aun cuando Yilmaz et al. (2003), mencionan que la SE con cemento portland en la matriz sólida es mejor a mayor valencia del metal, en el caso del cromo no se mantiene la regla por la particularidad de que el cromo hexavalente no forma hidróxidos insolubles y por tanto desestabiliza los hidratos formados que confieren estabilidad y resistencia a la compresión al hormigón.

La determinación de la eficiencia de retención de los metales, calculada con la ecuación 1, es un parámetro que permite validar la retención de los metales en relación de la masa; en tanto que la prueba de lixiviación PECT es un criterio legal. La Fig. 3 muestra la tendencia de retención de metales en el hormigón y se observa que el plomo tiene la mayor afinidad con el hormigón a base de cemento portland con valores de retención superiores al 99\% en un rango de estudio de hasta 5.5\% de plomo en el hormigón. En tanto que para el níquel las eficiencias de retención se encuentran entre el 96 y 98\% presentando una disminución al incrementar su concentración en el hormigón, en el rango estudiado. Para el caso del cromo se aprecian eficiencias de retención entre el 30\% y $45 \%$ en el rango de estudio, no se realizó la determinación de cromo a 5.2\% debido a la ausencia de fraguado del hormigón. De acuerdo al comportamiento de la efiencia de retención en los otros metales y sabiendo que a concentración de $0.52 \%$ de cromo en el hormigón se obtiene una eficiencia Información Tecnológica Vol. - 20 N¹ - 2009 
de retención de $43.6 \%$ de cromo; se puede destacar la necesidad de evaluar la retención de este metal en un intervalo de concentraciones más bajo o realizar un cambio del estado de oxidación del cromo a trivalente para promover la formación de hidróxidos.

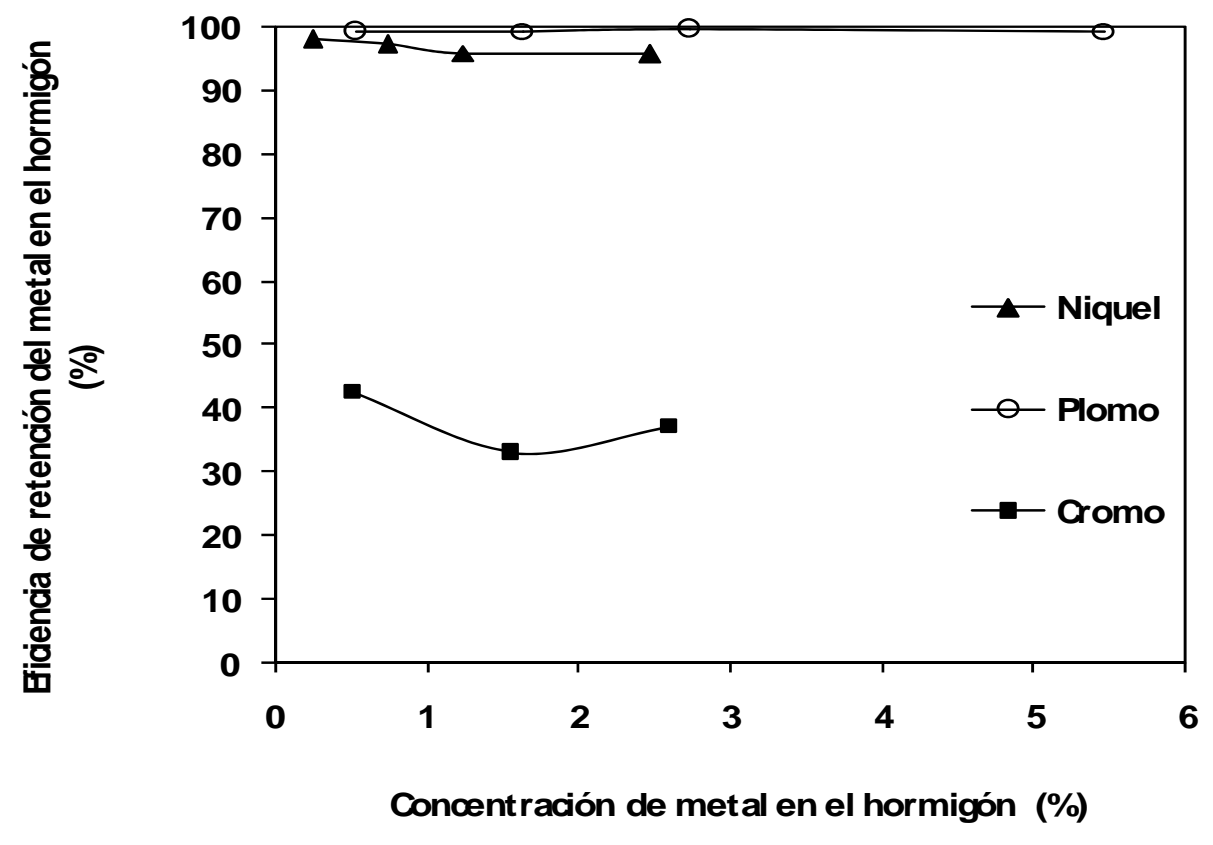

Fig. 3: Eficiencia de retención de los metales en el hormigón.

El objetivo de satisfacer los criterios de no-peligrosidad y uso estructural se logra cuando el hormigón producido no tiene características de peligrosidad por toxicidad con respecto a los valores de la NOM-052-SEMARNAT-2005 (2006), y que a estos valores de concentración en el hormigón, las resistencias a la compresión para los metales sean superiores a $14.7 \mathrm{~N} / \mathrm{mm}^{2}$ para uso en trabes y de $9.8 \mathrm{~N} / \mathrm{mm}^{2}$ para uso en pisos. En la Tabla 3 resumen los valores de concentración de metales en el hormigón que satisfacen ambos criterios.

Tabla 3: Máxima concentración permisible de metal en hormigón portland para no exceder norma de no-peligrosidad y tener uso estructural

\begin{tabular}{|c|c|c|c|}
\hline $\begin{array}{c}\text { Metal } \\
\text { pesado }\end{array}$ & $\begin{array}{c}\text { Concentración máxima de metal en el } \\
\text { hormigón portland para ser no } \\
\text { peligroso (\%) }\end{array}$ & $\begin{array}{c}\text { Resistencia a la } \\
\text { compresión } \\
\left(\mathrm{N} / \mathrm{mm}^{2}\right)\end{array}$ & Uso posible \\
\hline Plomo & $1.94 \%$ & 16.2 & Trabes y pisos \\
\hline Níquel & $0.43 \%$ & 12.8 & Pisos \\
\hline Níquel & $0.20 \%$ & 14.7 & Trabes y pisos \\
\hline Cromo & $0.02 \%$ & 28.5 & Trabes y pisos \\
\hline
\end{tabular}

El hormigón producido con metales a los valores de concentración máxima pueden tener aplicaciones comerciales, pudiendo ser utilizados en pisos, banquetas, calles y separaciones de autopistas como especificación constructiva baja o bien en trabes y cadenas como la especificación constructiva más alta.

\section{CONCLUSIONES}

El estudio realizado muestra que la técnica de solidificación-estabilización usando cemento portland CPO (cementación portland) con una formulación para una resistencia a la compresión de 29.4 $\mathrm{N} / \mathrm{mm}^{2}$ es una técnica que permite la inclusión de metales en el hormigón. Los resultados de laboratorio demuestran la factibilidad técnica del hormigón obtenido para usos en aplicaciones de construcción en función de sus valores de resistencia a la compresión satisfaciendo los estándares de construcción en México. 
Las pruebas de lixiviación PECT realizadas demuestran que existen valores límites de concentración de cromo, níquel y plomo que permiten establecer a las muestras de hormigón como un material con característica de no peligrosidad por toxicidad con valores de retención de plomo superiores al $99 \%$ de níquel superiores al 95\%. Los resultados con cromo hexavalente no son aceptables por lo que no se puede considerar a la cementación portland como una técnica no apta para este metal, sería conveniente el estudio como cromo trivalente, el cuál precipita como hidróxido metálico, para mejorar la retención y disminuir la interferencia en el fraguado del hormigón.

Lo anterior permite establecer los criterios máximos de inclusión de níquel y plomo en solución en la fabricación de hormigón que pueda ser utilizado comercialmente y de esta manera colaborar en la solución de problemas de contaminación del medio ambiente. Se puede ver a esta técnica como una alternativa para el tratamiento de corrientes líquidas con metales de la industria; también puede ser usada para disposición final de estos metales en rellenos sanitarios existentes en muchas ciudades y no en confinamientos controlados de residuos peligrosos, disminuyendo adicionalmente los costos de disposición.

\section{REFERENCIAS}

Al-Tabbaa, A. y S. Prose; Treatment Study for In-situ Stabilization/Solidification of Soil Contaminated with Methylene Blue, Environ. Technol.: 17(2), 191-197 (1996).

Aubert, J.E., B. Husson y N. Sarramone; Utilization of Municipal Solid Waste Incineration (MSWI) Fly Ash in Blended Cement. Part 1: Processing and Characterization of MSWI Fly Ash, J. Haz. Mat.: B136, 624-631 (2006).

Carmalin, S.A. y Swaminathan, K; Leaching of Metals on Stabilization of Metal Sludge Using Cement Based Materials, Journal of Env. Science: 17(1), 115-118 (2005).

Culliname, M.J., L.W. Jones y P.G. Malone; Handbook for Stabilization/Solidification of Hazardous Wastes, EPA/540/2-86-001, USEPA, USA (1986).

Fernández-OImo, I., C. Lasa y A. Irabien; Modeling of Zinc Solubility in Stabilized/Solidified Electric Arc Furnace Dust, J. Haz. Mat.: 144, 720-724 (2007).

Fernandez-Olmo, I., E. Chacón y A. Irabien; Leaching Behavior of Lead, Chromium (III) and Zinc in Cement/Metal Oxide Systems, Jour. Environ. Eng.: 129(6), 532-538 (2003).

Glasser, F.F.; Fundamental Aspects of Cement Solidification and Stabilisation, J. Haz. Mat.: 52, 151170 (1997).

Klich, I. y otros tres autores; Mineralogical Alterations that Affect the Durability and Metals Containment of Aged Solidified and Stabilized Wastes, Cement and Concrete Research: 29, 14331440 (1999).

Malviya, R. y R. Chaudhary; Leaching Behavior and Immobilization of Heavy Metals in Solidified/Stabilized Products. Journal of Hazardous Materials: B137, 207-217 (2006).

Minjo, V. y otros cuatro autores; Compositional Changes in Cement-Stabilized Waste During Leach Tests-Comparison of SEM/EDX Data with Predictions from Geochemical Speciation Modeling, Journal of Colloid and Interface Science: 280, 465-477 (2004).

NMX-AA-SCFI-2001 (2001): Secretaría de Economía, Determinación de Metales por Absorción Atómica en Aguas Naturales, Potables, Residuales y Residuales Tratadas-Método de Prueba, 1-52, México (2001). 
NMX-C-414-ONNCCE-2004: Organismo Nacional para la Normalización y Certificación de la Construcción y Edificación, Industria de la Construcción-Cementos Hidráulicos-Especificaciones y Métodos de Prueba, 1-10, México (2004).

NOM-052-SEMARNAT-2005: Secretaría de Medio Ambiente y Recursos Naturales, Norma Oficial Mexicana que Establece las Características, el Procedimiento de Identificación, Clasificación y los Listados de los Residuos Peligrosos, 1-32, México (2006).

NOM-053-SEMARNAT-1993: Secretaría de Medio Ambiente y Recursos Naturales, Norma Oficial Mexicana que Establece el Procedimiento para Llevar a Cabo la Prueba de Extracción para Determinar los Constituyentes que Hacen a un Residuo Peligroso por su Toxicidad al Ambiente, 122, México (2003).

NOM-127-SSA1-1994: Secretaría de Salud, Agua para Usos y Consumo Humano-Límites Permisibles de Calidad y Tratamientos a que debe Someterse el Agua para su Potabilización, 1-7, México (2000).

Paria, S. y P.K. Yuet; Solidification-Stabilization of Organic and Inorganic Contaminants Using Portland Cement: A Literature Review. Environ. Rev.: 14, 217-255 (2006).

Pellegrini, F. y otros cuatro autores; Sorption and Desorption of $\mathrm{Cd}$, Co, Cu, Ni and $\mathrm{Zn}$ from Carbonated Portland Cement, Advances in App. Ceramics: 105(4), 185-190 (2006).

Polletini, A., R. Pomi y M. Valente; Remediation of Heavy Metal-Contaminated Soil by Means of Agglomeration. Journal of Environmental Science and Health: A39, 999-1010 (2004).

Shi, C. y R. Spence; Designing of Cement-Based Formula for Solidification/Stabilization of Hazardous, Radioactive and Mixed Wastes, Crit. Rev. Env. Sci. Technol.: 34, 391-417 (2004).

Silveira, B.I. y otros tres autores; Effectiveness of Cement-Based Systems for Stabilization and Solidification of Spent Pot Liner Inorganic Fraction, J. Haz. Mat.: B98, 183-190 (2003).

Tchobanoglus, G., H. Theisen y S. Vigil; Integrated Solid Waste Management: Engineering Principles and Management Issues, Mc Graw Hill, Boston (2003).

Udoeyo, F.F. y otros tres autores; Potencial of Wood Waste Ash as an Additive in Concrete, Journal of Materials in Civil Eng.: 18(4), 605-611 (2006).

USEPA; Treatment Technologies for Site Cleanup: Markets and Technology Trends, EPA-542-R-96005, USEPA, USA (2004).

Van der Sloot, H.A.; Characterization of the Leaching Behavior of Concrete Mortars and of CementBased Wastes with Different Waste Loading for Long Term Environmental Assessment, Waste Management: 22, 181-186 (2002).

Yilmaz, O., U. Kahraman y E. Cokca; Solidification/Stabilization of Hazardous Wastes Containing Metals and Organic Contaminants, J. Environ. Eng.: 129(4), 366-376 (2003).

Zhang, L. y otros tres autores; Effects of Sucrose and Sorbitol on Cement-Based Stabilization/Solidification of Toxic Metal Waste, J. Haz. Mat.: 151, 490-498 (2008). 